\title{
Nasopharyngeal Cancer with Temporomandibular Disorder and Neurologic Symptom: A Case Report
}

\author{
Jung-Hun Hong, Jeong-Seung Kwon, Hyung-Joon Ahn, Seong-Taek Kim, Jong-Hoon Choi \\ Department of Orofacial Pain and Oral Medicine, College of Dentistry, Yonsei University, Seoul, Korea
}

Received January 4, 2014

Revised January 14, 2014

Accepted January 23, 2014

Correspondence to:

Jong-Hoon Choi

Department of Orofacial Pain and Oral

Medicine, Dental Hospital, College

of Dentistry, Yonsei University, 50,

Yonsei-ro, Seodaemun-gu, Seoul

120-752, Korea

Tel: +82-2-2228-3113

Fax: +82-2-393-5673

E-mail: jhchoij@yuhs.ac
Nasopharyngeal cancer is malignant tumor of nasopharyngeal area that is characterized of lymphadenopathy, pain, otitis media, hearing loss and cranial nerve palsy and may present symptoms similar to temporomandibular disorder such as facial pain and trismus. In this case, the patient with symptoms similar to temporomandibular disorder after surgery for otitis media presented with facial paresthesia and masticatory muscle weakness. Examinaion of trigemimal nerve was shown sensory and motor abnormaility. The patient was referred to a neurologist. Nasopharyngeal cancer was suspected on computed tomography and magnetic resonance imaging and was confirmed by biopsy. If the patient presenting with paresthesia and muscle weakness the cranial nerve examination should be performed regardless of typical temporomandibular disorder symptom. The neurologic symptom can be caused by neoplasm such as brain tumor and nasopharyngeal cancer. Nasopharyngeal cancer on rosenmuller fossa can develop otitis media. Therefore, the patient with otitis media history should be consulted to otorhinolaryngologist to examin the nasopharyngeal area.

Key Words: Muscle weakness; Nasopharyngeal neoplasms; Nasopharynx; Otitis media; Temporomandibular joint disorders

\section{INTRODUCTION}

The facial pain and trismus similar to symptom of temporomandibular disorder (TMD) can caused by various disease such as otorhinolaryngolgic disease, nerve and vascular disease, tumor, inflammation and infection. ${ }^{1)}$

\section{CASE REPORT}

A 30-year-old woman presented with dull sensation on left preauricular area, masseter, chin and half of tongue, headache and masticatory muscle weakness. Trismus was developed after surgery for otitis media performed 3 weeks ago. She went to an orthopedic surgean and was subscribed the antiinflammatory drug and muscle relaxant for 3 days. The trismus relieved but the headache and muscle weakness started 2 weeks ago. She could not fall asleep well because of left temporal headache relieved by pain killer. One week ago, paresthesia, tinnitus and masticatory muscle weakness that caused weak contact on left premolars and molars started. Two days ago, the area of paresthesia enlarged.

Orofacial examination was performed. There was tenderness to palpation on left temporomandibular joint, masseter and temporalis and both sternocleidomastoid muscle, trapezius and splenius capitis. Only right first and second molars occluded. Active mouth opening was $38 \mathrm{~mm}$ with pain on left masseter. On trigeminal nerve examination, sensation to light touch and pin prink test on left cheek was about ten percent compared to right side. Two point discrimination and pain threshold was slightly increased. Brush direction test was normal. There was weak contraction on left masseter during clenching. There was no significant pathologic bony change on transcranial and panoramic $\mathrm{X}$-ray.

The patient was diagnosed with trigeminal neuropathy temporomandibular joint arthralgia and myofascial pain. She was consulted to neurologist to rule out trigeminal neuropathy due to brain tumor. Magnetic resonance imaging and computed tomography scan were performed and

Copyright (c) 2014 Korean Academy of Orofacial Pain and Oral Medicine. All rights reserved. 

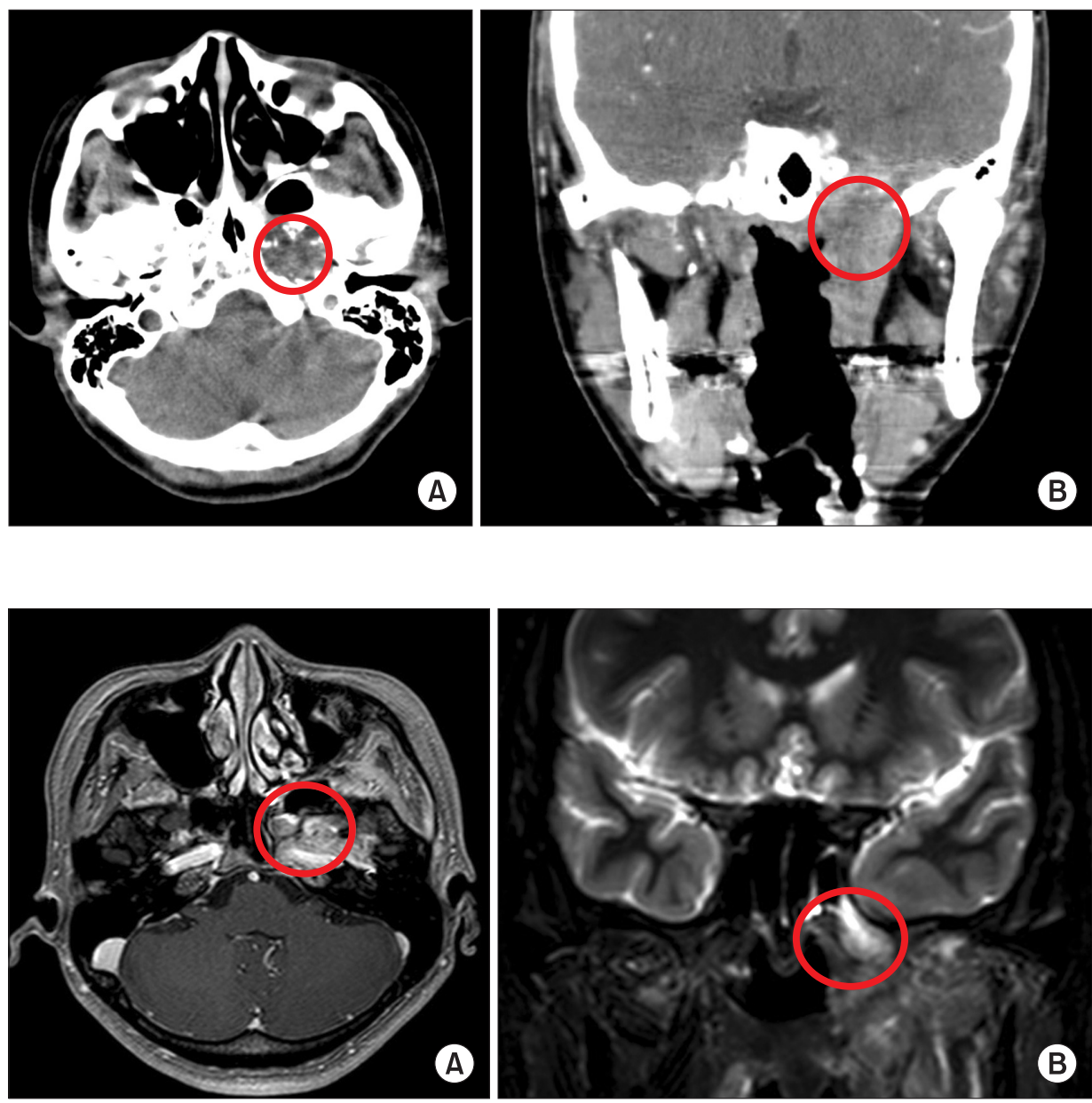

Fig. 1. Computed tomography scan image. The circles indicate the nasopharyngeal lesion. (A) Coronal view. (B) Frontal view.
Fig. 2. Magnetic resonance imaging image. The circles indicate the nasopharyngeal lesion. (A) Coronal view. (B) Frontal view. revealed the lesion in posterior nasopharynx including rosenmuller fossa (Figs. 1, 2). Nasopharyngeal cancer was suspected and biopsy confirmed adenoid cystic carcinoma. After that, she had recieved chemotherapy.

\section{DISCUSSION}

TMD can be caused by otorhinolaryngologic disease, nerve and vascular disease, tumor, inflammation and infection. The case that nasopharyngeal cancer caused trismus, pain and dull sensation similar to TMD symptoms was reported. ${ }^{2)}$ History taking is most important to differentially diagnose.

Common sign and symptom of nasopharyngeal cancer are mass of neck (cervical node metastases), blood stained saliva or sputum, headache, facial pain, nasal obstruction with epistaxis, otitis media, hearing loss, unilateral tinnitus, cranial nerve (VI, V, XII, IX, X) palsies. ${ }^{3)}$ In this case, she had history of otitis media, headache, cranial nerve palsies, tinnitus, and facial pain. Otitis media is often associated with nasopharyngeal cancer because of anatomical relationship. Rosenmuller fossa is common area to develop nasopharyngeal cancer ${ }^{4)}$ and close to the opening of auditory tube. If the enlarged tumor block the opening auditory tube which makes pressure control in ear, otitis media can be caused.

In summary, nasopharyngeal cancer may make TMD mimic symptoms. Consultation to Department of Otorhinolaryngology should be needed when presenting sign and symptom of nasopharyngeal cancer. But, cranial nerve examination is required when patient complain neurologic symptoms. ${ }^{5}$ We should keep in mind that through history taking and proper consultation leads the early diagnosis and proper treatment.

\section{CONFLICT OF INTEREST}

No potential conflict of interest relevant to this article was reported. 


\section{REFERENCES}

1. The American Academy of Orofacial Pain. Temporomandibular disorders. In: de Leeuw R, ed. Orofacial pain: guidelines for assessment, diagnosis, and management. 4th ed. Chicago: Quintessence Publishing; 2008. pp. 131-132.

2. Byun YS, Kim KS, Ahn HJ, Choi JH, Kwon JS. Temporomandibular disorder caused by nasopharyngeal cancer. Korean J Oral Med 2008;33:395-399.
3. Flint PW, Haughey BH, Lund VJ, et al. Cummings otolaryngology head and neck surgery. 5th ed. Philadelphia: Mosby Inc.; 2010. pp. 1348-1357.

4. Loh LE, Chee TS, John AB. The anatomy of the Fossa of Rosenmuller--its possible influence on the detection of occult nasopharyngeal carcinoma. Singapore Med J 1991;32:154-155.

5. Okeson JP. Management of temporomandibular disorders and occlusion. 6th ed. Seoul: Daehan Narae Publishing Inc.; 2009. pp. 239-242. 